Jódar Reverte, M.; Paredes Ruiz, M.J.; Martínez González-Moro, I.; Ferrer López, V. (2021). Effects of Nordic Walking on the Aerobic Endurance of Older Adults. Revista Internacional de Medicina y Ciencias de la Actividad Física y el Deporte vol. 21 (84) pp. 561-572. Http://cdeporte.rediris.es/revista/revista83/artefectos1260.htm

DOI: https://doi.org/10.15366/rimcafd2021.83.009

\title{
ORIGINAL
}

\section{EFECTOS DE LA MARCHA NÓRDICA SOBRE LA RESISTENCIA AERÓBICA DE ADULTOS MAYORES}

\section{EFFECTS OF NORDIC WALKING ON THE AEROBIC ENDURANCE OF OLDER ADULTS}

\author{
Jódar Reverte, M.1; Paredes Ruiz, M.J.1; Martínez González-Moro, I.; Ferrer \\ López, $\mathrm{V}^{3}$ \\ 1 Grupo de Investigación Ejercicio Físico y Rendimiento Humano. Universidad de Murcia \\ (España)mariajodarreverte@gmail.com, mariajoseparedesruiz@gmail.com \\ 2 Titular de Universidad. Grupo de Investigación Ejercicio Físico y Rendimiento Humano. \\ Universidad de Murcia (España) igmartgm@um.es \\ 3 Profesor Universidad de Murcia. Grupo de Investigación Ejercicio Físico y Rendimiento \\ Humano. Universidad de Murcia (España) vferrer@um.es
}

AGRADECIMIENTOS Y FINANCIACIÓN: Agradecemos la colaboración de la asociación Nordic Walking Murcia. Así mismo, este estudio ha sido financiado por el Grupo de Investigación Ejercicio Físico y Rendimiento Humano de la Universidad de Murcia.

Código UNESCO I UNESCO code: 9915 Medicina del Deporte / Sports Medicine

Clasificación Consejo de Europa / Council of Europe Classification: 11. Medicina del Deporte / Sports Medicine

Recibido 23 de junio de 2019 Received June 23, 2019

Aceptado 26 de octubre de 2019 Accepted October 26, 2019

\section{RESUMEN}

Introducción: El Objetivo fue evaluar los efectos sobre la resistencia aeróbica de un programa de ejercicio físico basado en la marcha nórdica para la mejora de la condición física en adultos mayores. Metodología: La población se compuso de 29 sujetos, los cuales realizaron un programa de marcha nórdica de doce semanas de duración, con un mínimo de dos sesiones semanales. Se llevaron a cabo dos mediciones; una previa y otra posterior, mediante prueba de esfuerzo. Resultados: La población mostró mejoras significativas en cuanto a la intensidad alcanzada durante la prueba de esfuerzo. Así mismo se observaron 
aumentos significativos $(p<0,05)$ en las variables consumo de oxígeno y frecuencia cardíaca. Conclusiones: El programa de marcha nórdica ha aumentado la condición física de nuestra población, encontrando mejoras a nivel cardiorrespiratorio tanto en hombres como mujeres.

PALABRAS CLAVE: ergoespirometría, marcha nórdica, capacidad funcional, ancianos, resistencia aeróbica.

\section{ABSTRACT}

Introduction: The objective was to evaluate the effects on aerobic endurance of a physical exercise program based on Nordic walking for the improvement of physical condition in older adults. Methodology: The population consisted of 29 subjects, the results of a twelve-week Nordic walking program, with a minimum of two weekly sessions. Two measurements were carried out; a previous and a subsequent one, through an effort test. Results: The population showed improvements in the intensity reached during the stress test. Likewise, significant increases $(p<0.05)$ were observed in the variables oxygen consumption and heart rate. Conclusions: The Nordic walking program has increased the physical condition of our population, finding improvements at the cardiorespiratory level in both men and women.

KEYWORDS: ergospirometry, Nordic walking, functional capacity, elderly, aerobic endurance.

\section{INTRODUCCIÓN}

La Marcha Nórdica (MN) es un deporte emergente que tiene su origen en Finlandia en 1930, sin embargo, esta práctica no se consolidó como la conocemos a día de hoy hasta finales del siglo $X X X^{1}$. Actualmente se desconoce el número de practicantes, aunque es un hecho que su práctica se ha extendido a nivel mundial ${ }^{2}$.En España, esta disciplina está regulada y coordinada por la Federación Española de Deportes de Montaña y Escalada (FEDME)².

Esta actividad consiste en caminar con unos bastones diseñados específicamente para ello. De este modo se consigue implicar toda la musculatura del tren superior e inferior, así mismo se reduce la carga en las articulaciones, rodillas, tobillos, cadera. El uso de estos bastones hace que la persona incline el cuerpo ligeramente hacia delante, además de exigir una coordinación contra-lateral de manos y pies ${ }^{3}$.

Numerosos estudios consideran la marcha nórdica como una actividad fácil, segura y saludable ${ }^{4,5}$. Por lo que además de fines competitivos se emplea como base de planes de entrenamiento para la mejora de la salud y de la condición física tanto en poblaciones específicas como en sujetos sanos ${ }^{3,6}$. 
Según la normativa de la FEDME las competiciones suelen hacerse sobre recorridos de una longitud entre 10 y $42 \mathrm{~km}^{7}$; así, por el tipo de ejercicio que se realiza, por el tiempo que duran sus sesiones y por las características metabólicas predominantes, podemos considerar que es un deporte de resistencia aeróbica ${ }^{6}$. Por tanto, la prueba médico-deportiva más adecuada para la valoración de sus participantes, tanto desde el punto de vista de salud y adaptación como de rendimiento es la ergoespirometría ${ }^{8}$. La ergometría estudia la respuesta del corazón ante el ejercicio físico mediante el análisis electrocardiográfico ${ }^{9,10}$. Esta prueba se emplea para el diagnóstico, seguimiento y prevención de la cardiopatía isquémica ${ }^{11,12}$ y del rendimiento de los deportistas ${ }^{13,14}$. Si a esto se añade el estudio espirométrico con la cuantificación de los gases respiratorios y se obtienen los valores de consumo máximo de oxígeno, cociente respiratorio y umbrales ventilatorios se pueden aportar datos sobre la adaptación al ejercicio que son utilizables para valorar el rendimiento de estos deportistas y la planificación del entrenamiento ${ }^{8}$. Por tanto, los valores obtenidos mediante la prueba ergoespirométrica pueden emplearse en diferentes planes de entrenamiento adecuándolos a las distintas capacidades físicas de cada sujeto ${ }^{15}$.

Cada vez son más los estudios que analizan los efectos de un programa de MN en poblaciones concretas, como pueden ser sujetos con diabetes ${ }^{16}$, sobrepeso $\mathrm{u}$ obesidad ${ }^{17},{ }^{18}$, enfermedad de Parkinson ${ }^{19,}{ }^{20}$, enfermedad pulmonar obstructiva crónica ${ }^{21,22}$, enfermedades cardiovasculares ${ }^{23,24,25}$, entre otros. La mayoría de los autores coinciden en que la $\mathrm{MN}$ es una actividad bien tolerada y que puede servir para contrarrestar los aspectos negativos asociados a la inactividad, ayudando de este modo a mantener un óptimo rendimiento general $^{26}$. Algunos de ellos se han realizado empleando la ergometría con análisis de gases para la evaluación de la respuesta fisiológica del organismo ante tal actividad 27,28 . Por ello la realización de este estudio puede aportar conocimiento sobre la recomendación de esta actividad en adultos mayores para la mejora y el mantenimiento de la salud.

\section{OBJETIVO}

Evaluar los efectos sobre la resistencia aeróbica de un programa de entrenamiento de marcha nórdica para la mejora de la condición física en adultos mayores.

\section{MATERIAL Y MÉTODO}

\section{Sujetos}

La población la formaron 26 sujetos 9 varones y 17 mujeres; con una edad media de $50.4 \pm 5.8$ años. Los participantes se inscribieron de forma voluntaria a través de la Federación de Montañismo de la Región de Murcia. Se incluyeron sujetos mayores de 40 años. Para determinar la exlusión de los participantes se realizó un cuestionario de antecedentes en salud para detectar a los participantes que 
debido a una enfermedad, alteración y/o lesión fueran incapaces de realizar el programa de entrenamiento y/o la prueba de esfuerzo. Previamente a la recogida de datos se informó a los participantes de los objetivos y procedimientos del estudio, todos ellos firmaron el correspondiente documento de consentimiento informado. Además, el estudio fue aprobado la Comisión de Ética de la Investigación de la Universidad de Murcia.

\section{Diseño}

Se trata de un estudio de carácter observacional, descriptivo, prospectivo. A cada participante se le realizaron dos evaluaciones, una antes del programa de entrenamiento y otra después de este. Cada evaluación consistía en la recogida de datos antropométricos, composición corporal y prueba de esfuerzo con análisis de gases.

La duración del programa de entrenamiento fue de 12 semanas consecutivas. Este programa fue el que habitualmente realiza el club organizador sin que se modificara para el presente estudio, fue diseñado y controlado por instructores experimentados de la FEDME. Previamente los instructores adiestraron a los participantes en la técnica de la MN, la postura a adoptar y el manejo de los bastones. Se realizaban dos sesiones semanales (martes y jueves) de 90 minutos de duración en circuito urbano y una salida mensual (sábado) por circuito extraurbano con 150 minutos. Cada sesión tiene una parte inicial y otra de vuelta a la calma con ejercicios de estiramientos y una central basada en la realización de un recorrido con una longitud entre 7-10 km los urbanos y 10-20 $\mathrm{Km}$ los extraurbanos. Durante los circuitos cada participante adoptaba su ritmo y velocidad a su capacidad.

En ambas valoraciones se siguió el mismo procedimiento. Tras las mediciones antropométricas (peso, talla y contornos de cintura y cadera) y la exploración cardiovascular basal (anamnesis, auscultación, presión arterial y electrocardiograma en reposo), a cada sujeto se le realizó una prueba de esfuerzo máxima sobre un tapiz rodante modelo Run 7411 (Runner®) con el registro continuo de las 12 derivaciones estándar del electrocardiograma (electrocardiógrafo Cardioline $\AA$, modelo Click ECG) y de la presión arterial cada dos minutos (Metronik BL-6®). Mediante el analizador de gases. Metalyzer® 3 B de Cortex se realizó el estudio de gases espirados, a partir del consumo máximo de oxígeno, el cociente respiratorio y la ventilación máxima.

Se utilizó un protocolo de Bruce modificado en rampa con una duración máxima de 15 minutos en el que la prueba se iniciaba con una velocidad de 1,9 Km/hora aumentando progresivamente la pendiente y/o lay/o la velocidad hasta llegar a lo $8 \mathrm{Km} / \mathrm{h}$ y $18 \%$ de pendiente. Durante la misma se registró la frecuencia cardíaca cada minuto y la presión arterial cada dos minutos durante el esfuerzo y a los minutos 1, 3 y 5 de recuperación. Estas mediciones han sido realizadas por un equipo conformado por un médico especialista en Ciencias del Deporte y una enfermera. 
La prueba finalizaba cuando el sujeto llegaba al máximo de su capacidad y lo indicaba alzando el brazo, en ese momento se iniciaba el periodo de recuperación. De igual modo se anotaba el motivo de finalización de la prueba. Para establecer la maximalidad de la prueba se comprueba que se supera el 1.1 en el cociente respiratorio y el $85 \%$ de la frecuencia cardíaca máxima prevista para su edad.

\section{Análisis estadístico}

Se utilizó el programa SPSS versión 24. Las variables cuantitativas se presentan mediante su media y desviación típica y las variables cualitativas con sus frecuencias absolutas y porcentajes. Previamente se determinó la normalidad de las distribuciones con el test de Shapiro Wilk y se han comparado las medias de variables independientes mediante la prueba de los rasgos de Wilcoxon y las relacionadas con la $T$ pareada. La relación entre variables cuantitativas se ha realizado con la correlación de Pearson. Los análisis se realizaron con un intervalo de confianza del $95 \%$ y $\mathrm{p} \leq 0,05$. El Índice de masa corporal se ha obtenido a partir de la fórmula masa corporal/ talla al cuadrado y la masa grasa relativa mediante el procedimiento de Woolcott y Bergman ${ }^{29}$, a partir de la altura y el perímetro de cintura, empleando la ecuación ajustada en función del género. Para el análisis de los datos se dividió a la población en dos grupos según su experiencia previa en $\mathrm{MN}$, veteranos $(n=9)$ con más de dos años de experiencia y noveles $(n=17)$, sin experiencia.

\section{RESULTADOS}

En la tabla I mostramos las características antropométricas de ambos grupos, separados por sexos.

Tabla I.- Características antropométricas

\begin{tabular}{ccccc}
\hline & \multicolumn{2}{c}{ Varones } & \multicolumn{2}{c}{ Mujeres } \\
\hline & $\begin{array}{c}\text { Veteranos } \\
(\mathrm{n}=4)\end{array}$ & $\begin{array}{c}\text { Noveles } \\
(\mathrm{n}=5)\end{array}$ & $\begin{array}{c}\text { Veteranas } \\
(\mathrm{n}=8)\end{array}$ & $\begin{array}{c}\text { Noveles } \\
(\mathrm{n}=12)\end{array}$ \\
\hline Edad (años) & $52,2 \pm 8,9$ & $48,6 \pm 4,2$ & $49,7 \pm 6,2$ & $51,1 \pm 4,3$ \\
\hline Talla $(\mathrm{cm})$ & $176,32 \pm 3,93$ & $180,02 \pm 5,21$ & $162,60 \pm 4,33$ & $164,71 \pm 5,43$ \\
\hline Masa corporal $(\mathrm{Kg})$ & $71,90 \pm 2,73$ & $88,46 \pm 4,98$ & $67,66 \pm 6,71$ & $67,38 \pm 12,26$ \\
\hline Cintura $(\mathrm{cm})$ & $88,75 \pm 4,99$ & $99,90 \pm 6,06$ & $91,93 \pm 6,21$ & $90,58 \pm 11,47$ \\
\hline IMC $($ Kg/m2) & $23,15 \pm 1,50$ & $27,40 \pm 2,23$ & $25,60 \pm 2,28$ & $24,79 \pm 3,80$ \\
\hline Masa grasa relativa $(\%)$ & $24,17 \pm 2,14$ & $27,84 \pm 2,52$ & $40,48 \pm 2,60$ & $39,20 \pm 3,85$ \\
\hline
\end{tabular}


En mujeres no existen diferencias significativas entre veteranas y noveles. En cambio, los hombres sin experiencia presentan valores significativamente más altos que los veteranos en cuanto a la masa corporal $(t=5,912 p=0,001)$, contorno de cintura $(t=2,952 p=0,021)$, e IMC $(t=3,236 p=0,014)$, indicando que para una talla similar poseen más masa grasa.

Tres de los sujetos, todos ellos varones, consiguieron realizar, en la primera valoración, el protocolo completo de la prueba de esfuerzo establecido en 15 escalones de un minuto cada uno, con una velocidad máxima de $8 \mathrm{~km} / \mathrm{hora}$ y una pendiente del 18\%, estos en la segunda evaluación volvieron a completar el protocolo. En la tabla II mostramos los valores obtenidos por estos participantes al finalizar ambas valoraciones.

Tabla II.- Comparación de las características ergométricas de los sujetos que completaron el protocolo en ambas evaluaciones, mediante el test de Wilcoxon.

\begin{tabular}{ccccc}
\hline $\mathrm{n}=3$ & Pre-programa & Post-programa & $\mathrm{t}$ & Sig. \\
\hline FC (lat/min) & $165,66 \pm 24,90$ & $168,00 \pm 27,49$ & 593 & $>0,05$ \\
\hline FC $\%$ & $97,24 \pm 14,50$ & $98,62 \pm 16,11$ & 593 & $>0,05$ \\
\hline RER & $1,06 \pm 0,06$ & $1,03 \pm 0,07$ & 180 & $>0,05$ \\
\hline VO2 $(\mathrm{ml} / \mathrm{Kg} / \mathrm{min})$ & $33,33 \pm 3,05$ & $34,33 \pm 5,03$ & 1000 & $>0,05$ \\
\hline Ventilación $(\mathrm{I} / \mathrm{min})$ & $71,95 \pm 8,69$ & $74,65 \pm 9,66$ & 1000 & $>0,05$ \\
\hline
\end{tabular}

La tabla III muestra la comparación de los valores medios de cada una de las variables entre ambas valoraciones, separados por sexo, en aquellos sujetos que no completaron el protocolo de evaluación en la primera evaluación y que por tanto eran susceptibles de mejorarlo en la segunda. 
Tabla III.- Comparación de las características ergométricas de los sujetos que no completaron el protocolo en la primera evaluación.

\begin{tabular}{|c|c|c|c|c|c|c|c|c|}
\hline & \multicolumn{4}{|c|}{ Varones $(n=6)$} & \multicolumn{4}{|c|}{ Mujeres $(n=20)$} \\
\hline & Pre & Post & $\mathrm{t}$ & Sig. & Pre & Post & $\mathrm{t}$ & Sig. \\
\hline FC (lat/min) & $169,3 \pm 9,1$ & $174 \pm 8,02$ & 2,340 & 0,066 & $164,7 \pm 15,9$ & $172,1 \pm 15,8$ & 2,969 & 0,008 \\
\hline FC \% & $99,93 \pm 3,64$ & $102,72 \pm 3,62$ & 2,406 & 0,061 & $97,3 \pm 8,92$ & $101,64 \pm 7,99$ & 2,975 & 0,008 \\
\hline $\begin{array}{l}\text { Velocidad } \\
(\mathrm{Km} / \mathrm{h})\end{array}$ & $6,53 \pm 0,53$ & $7,06 \pm 0,56$ & 5,060 & 0,004 & $5,97 \pm 0,93$ & $6,47 \pm 0,83$ & 3,926 & 0,001 \\
\hline Pendiente (\%) & $16 \pm 0,89$ & $16,83 \pm 0,75$ & 5,000 & 0,004 & $15,05 \pm 1,43$ & $15,88 \pm 1,32$ & 3,570 & 0,003 \\
\hline Tiempo (min) & $11,27 \pm 1,38$ & $12,486 \pm 1,53$ & 4,379 & 0,007 & $1,10 \pm 0,06$ & $1,13 \pm 0,06$ & 1,158 & 0,265 \\
\hline RER & $1,13 \pm 0,04$ & $1,15 \pm 0,09$ & 0,442 & 0,677 & $9,87 \pm 2,03$ & $11,12 \pm 2,10$ & 4,030 & 0,001 \\
\hline $\begin{array}{l}\mathrm{VO} 2 \\
(\mathrm{ml} / \mathrm{Kg} / \mathrm{min})\end{array}$ & $35,33 \pm 3,61$ & $37,83 \pm 3,43$ & 4,443 & 0,007 & $25,56 \pm 3,54$ & $27,81 \pm 3,53$ & 4,980 & 0,000 \\
\hline $\begin{array}{l}\text { Ventilación } \\
(1 / \min )\end{array}$ & $91,3 \pm 19,26$ & $104,3 \pm 29,11$ & 2,066 & 0,094 & $58,93 \pm 8,7$ & $64,63 \pm 6,94$ & 3,427 & 0,003 \\
\hline
\end{tabular}

Pre= Valoración previa al programa Post = Valoración tras el programa

Se observa un aumento significativo de la velocidad tanto en hombres como en mujeres $(t=5,06 p=0,004)$ y $(t=3,93 p=0,000)$, respectivamente. Además, la pendiente ascendió significativamente, casi un grado tanto en hombres $(t=5,00$ $p=0,004)$, como en mujeres $(t=3,57 p=0,000)$. Del mismo modo observamos un incremento significativo del tiempo, aumentando más de un minuto en ambos grupos. El consumo máximo de oxígeno es otra de las variables que asciende significativamente en ambos grupos, $(t=4,44 p=0,0007)$ en hombres y $(t=4,98$ $p=0,000)$ en mujeres. Además, en mujeres destaca el aumento significativo de la frecuencia cardíaca máxima $(t=-2,96 p=0,000)$ y del porcentaje de frecuencia cardíaca $(t=-2,97 p=0,000)$

\section{DISCUSIÓN}

El objetivo del presente estudio fue analizar el efecto de un programa de marcha nórdica de 12 semanas de duración sobre la resistencia aeróbica de adultos mayores. Los resultados obtenidos indican un aumento significativo de la intensidad alcanzada durante la prueba de esfuerzo posterior al programa de ejercicio, que se ha objetivado con aumentos significativos en el tiempo de esfuerzo y por tanto en la velocidad y pendiente máxima alcanzada. Ello se ha acompañado con valores más altos en el consumo máximo de oxígeno.

Estudios anteriores al nuestro analizan los efectos de la marcha nórdica sobrepoblaciones similares a la nuestra y con un número de sujetos parecido, pero con una media de edad más avanzada, en torno a los 60-70 años, todos ellos han sido sujetos sanos y en ambos se buscaba el efecto del ejercicio sobre capacidades funcionales ${ }^{30,31}$.

Hemos tenido en cuenta la experiencia previa en la práctica de marcha nórdica dividiendo a nuestra población en veteranos y noveles, esta separación no se 
ha visto en la bibliografía referente a la práctica de esta disciplina en adultos mayores, tampoco en las otras poblaciones con personas con diabetes ${ }^{16}$, hipertensión ${ }^{32}$, sobrepeso u obesidad ${ }^{17,}{ }^{18}$, enfermedades cardiovasculares ${ }^{23,24,}$ 25 , Parkinson ${ }^{19,20}$ o cáncer de mama ${ }^{33}$ que han practicado marcha nórdica. Se ha considerado esta división puesto que un nivel de experiencia previo puede hacer que los cambios no se produzcan o sean leves debido a la habituación al ejercicio.

Para evaluar la resistencia aeróbica empleamos el protocolo de Bruce modificado en rampa, diversos estudios utilizan instrumentos o pruebas diferentes como prueba de campo al aire libre ${ }^{30}$, test de Cooper $^{34}$ o test de la marcha de 6 minutos ${ }^{35}$. En nuestro trabajo, consideramos la realización de la prueba ergométrica en condiciones de laboratorio ya que un medio controlado supone mayor exactitud en los resultados obtenidos y aumenta la fiabilidad de las comparaciones.

El plan de entrenamiento llevado a cabo tuvo una duración de 12 semanas consecutivas, al igual que en otros estudios revisados $24,25,30,36$. Además se realizaron un mínimo de dos sesiones semanales y una sesión mensual extra de mayor duración. El número de sesiones es variable entre las diferentes publicaciones, algunos cuentan con dos $^{30}$, pero la mayoría realizan tres sesiones semanales ${ }^{34}, 37,38$. Aunque el número de sesiones semanales de nuestro programa es inferior al de otros estudios, los resultados muestran que algo más de dos sesiones resultan suficientes para inducir cambios positivos en esta población, a pesar de ello si se aumenta el número se obtendrían mayores beneficios.

Nuestros resultados muestran que la marcha nórdica proporciona beneficios a nivel cardiorrespiratorio. Tras doce semanas de entrenamiento la población mostró mejoras significativas en cuanto a la intensidad alcanzada durante la prueba de esfuerzo. Esto explica el incremento en las variables frecuencia cardíaca y consumo máximo de oxígeno en ambos grupos, puesto que el trabajo realizado fue mayor.

Hemos de tener en cuenta que llevamos a cabo la prueba con carácter de maximalidad. Por tanto, consideramos beneficioso un incremento de la frecuencia cardíaca debido al aumento del esfuerzo realizado en la segunda valoración. En cambio, otros estudios en los que la naturaleza del examen era submáximo ${ }^{30}$, encuentran beneficioso el descenso de la frecuencia cardíaca entre pruebas, teniendo en cuenta que no hay un aumento del esfuerzo entre ambas. Valoramos que el aumento del esfuerzo tras el programa de ejercicio tiene mayor influencia positiva sobre la condición física del individuo.

En cuanto al consumo de oxígeno, empleamos un analizador de gases con medición directa, por el contrario, otros autores usan métodos de estimación indirecta ${ }^{30}$. Consideramos que la medición directa proporciona mayor precisión a los resultados, ya que cuenta con una medición constante. De nuevo el 
consumo de oxígeno mostró un incremento significativo debido al aumento del trabajo realizado por los participantes.

Encontramos otros trabajos que han evaluado programas de entrenamiento de marcha nórdica en personas mayores ${ }^{30,31,34}$ los cuales apuntan que esta actividad tiene un efecto beneficioso sobre la capacidad de resistencia aeróbica de sus practicantes.

Dentro de las limitaciones del estudio, destaca que nuestra población cuenta con un alto componente motivacional, ya que buscan con la práctica de la marcha nórdica mejorar su condición física y estado de salud. Esto hace que sea difícil extrapolar nuestros resultados a otros colectivos.

En cuanto a la aplicabilidad práctica, consideramos que la información aportada por este estudio permite elaborar programas de marcha nórdica destinados a la mejora de la salud de adultos mayores. Para ello, se recomienda que previamente al programa de ejercicio se valore de forma individualizada la condición física de cada sujeto. De este modo, se conseguirá adecuar la intensidad del ejercicio en función de los objetivos deseados, adaptando este a las necesidades, preferencias y limitaciones del deportista.

\section{CONCLUSIONES}

Los resultados obtenidos muestran un incremento de la intensidad alcanzada durante la prueba de esfuerzo, reflejando mejoras a nivel cardiorrespiratorio en ambos grupos. Por lo tanto, podemos concluir que el programa de ejercicio en marcha nórdica ha aumentado la capacidad de resistencia aeróbica en nuestra población.

\section{REFERENCIAS BIBLIOGRÁFICAS}

1. Encarnación A. Análisis biomecánico de la Marcha Nórdica: efectos de la experiencia y de la velocidad de práctica sobre el patrón de presión plantar, las fuerzas de reacción del suelo y los niveles de impacto. [Tesis doctoral]. Valencia: Departamento de educación física y deportiva, Universidad de Valencia; 2012.

2. Federación Española de Deportes de Montaña y Escalada. Qué es la Marcha Nórdica [Internet]. [cited 2019 Feb 23]. Disponible en: http://www.fedme.es/index.php?mmod=staticContent\&IDf=420

3. Pérez-Soriano P, Encarnación-Martínez A, Aparicio-Aparicio I, Giménez JV, Llana-Belloch S. Nordic walking: a systematic review. Eur J Hum Mov. 2014; 33:26-45.

4. Vílchez Barrera ME, Calvo-Arencibia A. Evidencia científica de la marcha nórdica en Fisioterapia: revisión bibliográfica. Fisioterapia. 2016; 38(5):25164. 
5. Martínez Lemos RI, García O, Serrano V. Nordic Walking y salud: una revisión descriptiva. Cuadernos de Psicología del Deporte. 2011; 11 (2):11512.

6. Tschentscher M, Niederseer D, Niebauer J. Health benefits of nordic walking: A systematic review. Am J Prev Med. 2013; 44(1):76-84.

7. Federación Española de Deportes de Montaña y Escalada. Reglamento de competiciones de marcha nórdica FEDME. 2018. [Internet]. [cited2019 Abr 29]. Disponibleen:http://www.fedme.es/salaprensa/upfiles/1535_F_es.pdf

8. Arós F, Boraita A, Alegría E, Alonso ÁM, Bardají A, Lamiel R, et al. Guías de práctica clínica de la Sociedad Española de Cardiología en pruebas de esfuerzo. Rev Esp Cardiol. 2000; 53: 1063-1094.

9. Arena R. Detecting abnormal pulmonary hemodynamics with cardiopulmonary exercise testing. Med Sci Sports Exerc. 2011; 43:982.

10. Valdés Martín A, Rivas Estany DE, Tadiana D, Aguilar A, Lila D, Echevarria Sifontes A. Utilidad de la Ergoespirometría en el diagnóstico y evaluación de las enfermedades cardiovasculares. Rev Cubana Cardiol. 2016; 22(1).

11. Calderón FJ, Benito PJ, García A. Aplicación práctica de las pruebas de esfuerzo. Selección. 2002; 11(4):202-9.

12. Alegría Ezquerra E, Alijar de Guimerá M, Cordo Mollar JC, Chorro Gascó FJ, Pajarón López A. Utilidad de la prueba de esfuerzo y de otros métodos basados en el electrocardiograma en la cardiopatía isquémica crónica. Rev Española Cardiol. 1997; 50(1):6-14.

13. Ronconi M, Alvero-Cruz JR. Respuesta de la frecuencia cardíaca y consumo de oxígeno de atletas varones en competiciones de duatlón sprint. Apunts Med l'Esport. 2011; 46(172):183-8.

14. Álvarez R, Campos DC, Portes P, Rey R, Martín B. Análisis de parámetros fisiológicos en jugadores juveniles españoles de bádminton. Rev Int la Med y Ciencias la Act Fis y del Deport. 2016; 16(61):44-5.

15. Bouzas JC, Ottoline NM, Delgado M. Aplicaciones de la frecuencia cardíaca máxima en la evaluación y prescripción de ejercicio. Apunts Med l'Esport. 2010; 45: 251-8.

16. Gram B, Christensen R, Christiansen C, Gram J. Effects of nordic walking and exercise in type 2 diabetes mellitus: a randomized controlled trial. Clin J Sport Med. 2010; 20(5): 355-361.

17. Figard-Fabre H, Fabre N, Leonardi A, Schena F. Physiological and perceptual responses to Nordic walking in obese middle-aged women in comparison with the normal walk. Eur J Appl Physiol. 2010; 108(6):1141-51.

18. Hagner-Derengowska M, Kałużny K, Kochański B, Hagner W, Borkowska A, Czamara A, et al. Effects of Nordic Walking and Pilates exercise programs on blood glucose and lipid profile in overweight and obese postmenopausal women in an experimental, nonrandomized, open-label, prospective controlled trial. Menopause. 2015; 22(11):1215-23.

19. Cugusi L, Solla P, Serpe R, Carzedda T, Piras L, Oggianu M, et al. Effects of a Nordic Walking program on motor and non-motor symptoms, functional performance and body composition in patients with Parkinson's disease. Neuro Rehabilitation. 2015; 37(2):245-54. 
20. Monteiro EP, Franzoni LT, Cubillos DM, De Oliveira Fagundes A, Carvalho $A R$, Oliveira $H B$, et al. Effects of Nordic walking training on functional parameters in Parkinson's disease: a randomized controlled clinical trial. Scand J Med Sci Sports. 2017; 27(3):351-8.

21. Breyer M-K, Breyer-Kohansal R, Funk G-C, Dornhofer N, Spruit MA, Wouters $E F$, et al. Nordic Walking improves daily physical activities in COPD: a randomised controlled trial. Respir Res. 2010; 11(1):112.

22. Barberan-Garcia A, Arbillaga-Etxarri A, Gimeno-Santos E, Rodríguez DA, Torralba Y, Roca J, et al. Nordic walking enhances oxygen uptake without increasing the rate of perceived exertion in patients with chronic obstructive pulmonary disease. Respiration. 2015; 89(3):221-5.

23. Kocur P, Deskur-Śmielecka E, Wilk M, Dylewicz P. Effects of Nordic Walking training on exercise capacity and fitness in men participating in early, shortterm inpatient cardiac rehabilitation after an acute coronary syndrome - a controlled trial. Clin Rehabil. 2009; 23(11):995-1004.

24. Keast M-L, SlovinecD'Angelo ME, Nelson CRM, Turcotte SE, McDonnell LA, Nadler RE, et al. Randomized trial of Nordic walking in patients with moderate to severe heart failure. Can J Cardiol. 2013; 29(11):1470-6.

25. Spafford C, Oakley C, Beard JD. Randomized clinical trial comparing Nordic pole walking and a standard home exercise programe in patients with intermittent claudication. Br J Surg. 2014; 101(7):760-7.

26. Tschentscher M, Niederseer D, Niebauer J. Health benefits of nordic walking: A systematic review. Am J Prev Med. 2013; 44(1):76-84.

27. Porcari JP, Hendrickson TL, Walter PR, Terry L, Walsko G. The Physiological Responses to Walking with and without Power Poles ${ }^{\mathrm{TM}}$ on Treadmill Exercise. Res Q Exerc Sport. 1997; 68(2):161-6.

28. Sugiyama K, Kawamura M, Tomita H, Katamoto S. Oxygen uptake, heart rate, perceived exertion, and integrated electromyogram of the lower and upper extremities during level and Nordic walking on a treadmill. J Physiol Anthropol. 2013; 32(1):2.

29. Woolcott OO, Bergman RN. Relative fat mass (RFM) as a new estimator of whole-body fat percentage -A cross-sectional study in American adult individuals. Sci Rep. 2018; 8(1):1-11.

30. Morat T, Krueger J, Gaedtke A, Preuss M, Latsch J, Predel H-G. Effects of 12 weeks of Nordic Walking and XCO Walking training on the endurance capacity of older adults. Eur Rev Aging Phys Act. 2017; 14(1):16.

31. Virág A, Karóczi CK, Jakab Á, Vass Z, Kovács É, Gondos T. Short-term and long-term effects of nordic walking training on balance, functional mobility, muscle strength and aerobic endurance among Hungarian community-living older people: a feasibility study. J Sports Med Phys Fitness. 2015; 55(11):1285-92.

32. Launois P, Khoudeir A, Pujol V, García N, Fargas J, Mena J, et al. Does Nordic walking helps to improve exercise capacity and weight control in patients with resistant hypertension? Ann Phys Rehabil Med. 2018; 6.

33. Malicka I, Stefańska M, Rudziak M, Jarmoluk P, Pawłowska K, SzczepańskaGieracha J, et al. The influence of Nordic walking exercise on upper extremity strength and the volume of lymphoedema in women following breast cancer treatment. Isokinet Exerc Sci. 2011; 19(4):295-304. 
34. Takeshima N, Islam MM, Rogers ME, Rogers NL, Sengoku N, Koizumi D, Naruse A. Effects of nordic walking compared to conventional walking and band-based resistance exercise on fitness in older adults. J Spots Sci Med, 2013; 12(3), 422-430.

35. Ossowski ZM, Skrobot W, Aschenbrenner P, Cesnaitiene VJ, Smaruj M. Effects of short-term nordic walking training on sarcopenia-related parameters in women with low bone mass: A preliminary study. Clinlnterv Aging. 2016; 11:1763-71.

36. Prusik K, Kortas J, Prusik K, Mieszkowski J, Jaworska J, Skrobot W, et al. Nordic Walking Training Causes a Decrease in Blood Cholesterol in Elderly Women Supplemented with Vitamin D. Front Endocrinol. 2018;9:42.

37. Kortas J, Kuchta A, Prusik K, Prusik K, Ziemann E, Labudda S, et al. Nordic walking training attenuation of oxidative stress in association with a drop in body iron stores in elderly women. Biogerontology. 2017; 18(4):517-24.

38. Schiffer T, Knicker A, Hoffman U, Harwig B, Hollmann W, Strüder HK. Physiological responses to nordic walking, walking and jogging. Eur J Appl Physiol. 2006; 98(1):56-61.

Número de citas totales/Total references: 38 (100\%)

Número de citas propias de la revista/Journal's own references: 1 (2,63\%)

Rev.int.med.cienc.act.fís.deporte - vol. 21 - número 83 - ISSN: 1577-0354 